COMMENT. The prognostic value of video-EEG monitoring and estimation of risk of SUDEP in patients with refractory generalized seizures is demonstrated in this study. Patients at risk should receive more aggressive medical and possibly surgical therapy as well as close postictal observation and stimulation to minimize the occurrence of central inhibition, apnea and SUDEP. Dr LJ Hirsch, Neurological Institute, New York, $\mathrm{NY}$, in an editorial, recommends the use of multimodal ambulatory monitoring (respiration, oxygen saturation, CO2, EKG, and EEG) in epilepsy patients at risk of SUDEP. He cites a prospective, international study in Europe and Australia, using cardiorespiratory and EEG monitoring in 16 cases of SUDEP, reporting central shutdown and respiratory arrest as the primary mechanism of death. (Findings presented at recent European Congress of Epileptology). The quality of life of patients with epilepsy needs protection, but the increasing interest in SUDEP indicates the need for closer monitoring of patients at risk.

\title{
RISK FOR DEVELOPING EPILEPSY
}

Lifetime risk and cumulative incidence of epilepsy were examined among Rochester, MN, residents between 1960 and 1979, and are reported from Columbia University, New York; and Mayo Clinic. Rochester, MN. Among 412 individuals identified with incident epilepsy, lifetime risk was $1.6 \%$ to age 50 and $3.0 \%$ to age 80 ; cumulative incidence $0.9 \%$ to age $20,1.7 \%$ to age 50 and $3.4 \%$ to age 80 . Lifetime risk through 87 years of age increased over time from $3.5 \%$ in $1960-69$ to $4.2 \%$ in $1970-79$. One in 26 people will develop epilepsy during their lifetime. (Hesdorffer DC, Logroscino G, Benn EKT, Katri N, Cascino G, Hauser WA. Estimating risk for developing epilepsy. A population-based study in Rochester, Minnesota. Neurology January 2011;76:23-27). (Response and reprints: Dr DC Hesdorffer, Columbia University, 630 West $168^{\text {th }}$ St, P\&S Unit 16, New York, NY 10032. E-mail: dch5@ columbia.edu).

COMMENT. Lifetime risk forecasts the need for epilepsy services in a community. The greatest need for these services is in infants and the elderly. The incidence curve varies with age, with high rates in infants under 1 year of age, low rates from age 20 to 60 , and an increase from 60 to age 80 , a time period that is rapidly expanding in the general population. The authors estimate that 12 million individuals in the US will develop epilepsy in their lifetime.

\section{WEST SYNDROME REMISSION FOLLOWING ACUTE VIRAL INFECTION}

Researchers at Nihon University School of Medicine, Tokyo, Japan, report 11 children with intractable epilepsy (West syndrome in 6 and myoclonic seizures in 5) who showed clinical and electrographic improvement following acute viral infection. Seizure remission occurred following exanthem subitum in 5 patients, rotavirus gastroenteritis in 2 , measles in 2 , herpetic stomatitis in 1 , and common cold in 1 . In patients with West syndrome, salaam and/or tonic spasms resolved within 6 days after onset of viral infection, and hypsarrhythmia was modified and evolved to localized spikes. Myoclonic seizures resolved rapidly and completely and the EEG gradually normalized or improved. 